\title{
Periodic walking motion of a Humanoid robot based on human data
}

\author{
Anne Kalouguine ${ }^{1,2}$, Christine Chevallereau ${ }^{1}$, Sébastien Dalibard ${ }^{2}$ \\ and Yannick Aoustin ${ }^{1}$ \\ ${ }^{1}$ Ecole Centrale de Nantes, Université de Nantes, CNRS, LS2N, F-44000 \\ Nantes, France, e-mail: surname.namedIs2n. fr ${ }^{2}$ SoftBank Robotics, 43, \\ Rue du Colonel Pierre Avia 75015 Paris, e-mail: \\ surname.namelsoftbankrobotics.com
}

\begin{abstract}
Human walking has been intensely studied, but it is difficult to reproduce on humanoid robots that maintain awkward movements. Three main difficulties exist. (i) Different joint kinematics and size between humans and robots. (ii) A rolling motion of the foot which is often impossible to execute with humanoid robots that walk with their feet flat. (iii) A difference in the dynamic model of a robot compared to a human that makes a copy of a human movement lead to unstable walking. In order to take into account the first two difficulties, the specifications for reproducing human movements are adjusted. To ensure stability, a previously developed dynamic model called Essential Model is used. The zero moment point (ZMP) is imposed, and the horizontal evolution of the centre of mass (CoM) is computed to satisfy the ZMP.
\end{abstract}

Key words: Humanoid robot, Human-like walking, Center of Mass, Zero Moment point, Essential Model.

\section{Introduction}

Humanoid robots are complex mechatronic machines due to their numerous degrees of freedom, physical characteristics such as their weight, the limitations of their actuators, the unilateral constraints with the ground etc. To design walking motions is a complex challenge [1]. To overcome these difficulties, many researchers define the walking motions by using the linear inverted pendulum (LIP) model [2]. This model is efficient to obtain walking motions, but the resulting gait is not very human-like and the dynamic influence of the different bodies of the humanoid robot is not taken into account. Several approaches to imitate the human motion have been developed. For example a walking gait based on human-like virtual constraints has been investigated in [3] for the robot Nao. Sakka, who carried out a work about the imitation of human motion with Nao, also performed this type of study [4]. However, their approach does not consider the constraints on the ZMP trajectory, which are essential for stability. 
The purpose of this work is to design a periodic walking motion with single support (SS) and double support (DS) phases, which is based on the Essential model [5] for Romeo - a humanoid robot with $n=31$ generalized coordinates. The trajectory of the ZMP is imposed, the horizontal position of the CoM is free to adapt to the ZMP evolution. The CoM is thus computed from this ZMP evolution. The original contribution is that the remaining $n-2$ generalized coordinates are prescribed by using trajectories inspired from human walking data. The recorded human motions are approximated by sinusoidal functions of time.

The paper is outlined as follows. The main characteristics of human walking are presented in section 2. A reference walking motion based on human data is presented in section 3. This section highlights the necessity of the Essential model, which is then detailed in section 4 . The cyclic walking motion is stated in section 5. Numerical results are analyzed in section 6 . Section 8 offers our conclusions and perspectives.

\section{Study of human walking.}

Duration of different phases: Human walking can be decomposed according to important events that occur during the walking. A gait cycle consists of two steps. The duration of different phases is measured as a percentage of a cycle duration. The percentage of DS phase varies from 9 to $17 \%$ depending on the age and velocity of the human [6].

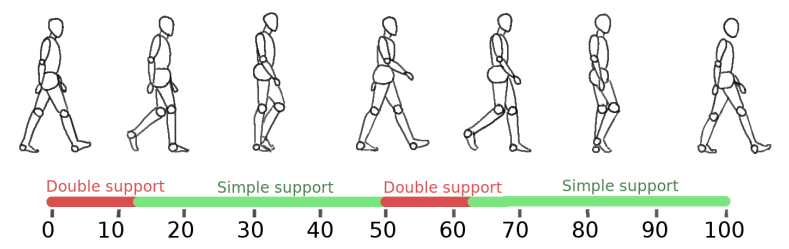

Fig. 1 SS and DS phases duration, measured as percentage of complete cycle.

Step placement: The step length and width vary widely depending on morphology and age. For a young healthy adult the step length varies widely (from 0.40 to $0.80 \mathrm{~m}$ for larger velocities), same as the step width (from 0.125 to $0.22 \mathrm{~m}$, with width decreasing for larger velocities) $[6,7]$.

CoM Trajectory: Human CoM trajectory is close to a sinus in longitudinal, transverse and vertical directions [6]. The magnitude and period of oscillations in transverse direction vary with speed [8]. In vertical direction the magnitude of the oscillations increases with velocity and is equal to about $2 \%$ of body height. 
ZMP trajectory: The ZMP (Zero Moment Point) goes from the heel to the tip of each foot [9], which corresponds to the rolling motion of the feet and the mobility of the human sole. The trajectory of the ZMP changes depending on the footwear of the human [10].

Swing foot motion: The motion of the swing foot can be separated in two components, the trajectory of the swing foot as a whole and the orientation of the sole. We observe nearly vertical landing and takeoff trajectories, with most of the horizontal movement performed in the middle of SS.

Trunk motion: The trunk, which represents $60 \%$ of the weight, has significant angular oscillations[11]: in the saggital plane, the amplitude is of about $2 \circ$ around the equilibrium position (which varies with the walking velocity but is typically between 5 and 13०, leaning forward). In the frontal plane, the oscillations amplitude varies from 3 to 60 depending on the velocity.

Hip motion: The oscillations of the hip and the basin allow to make bigger steps, to smoothen the trajectory of the CoM. The amplitude of the oscillations around the vertical axis is of about $10^{\circ}[6]$.

Arm swing: The arm swing in human locomotion is speculated to be useful to reduce the contact wrench on the support foot, as well as the global cost of walking [12], [13].

\section{Human trajectory and humanoid robot}

Once the human trajectories are found, they can be applied to a humanoid robot. However, that does not give a viable walking motion. Romeo is a prototype platform issued by company Softbank Robotics, see figure 3 a). It is $1 \mathrm{~m} 47$ tall, weighs $36 \mathrm{~kg}$ and features 31 degrees of freedom groups into the configuration vector $q$. The duration of the DS phase is chosen to be close to $12 \%$ of the cycle duration $2 \cdot T$, where $T=T_{D S}+T_{S S}, T_{D S}=0.15 \mathrm{~s}$ and $T_{S S}=0.60 \mathrm{~s}$ are the durations of the DS phase and SS phases. It is impossible to achieve a step size of $0.75 \mathrm{~m}$ as what is observed for humans, because the pelvic rotation and rolling motion of the stance foot is necessary for these larger steps, see [14]. It is necessary to adapt the parameters of trajectories for Romeo. The step width is chosen to be $0.20 \mathrm{~m}$ to satisfy a safe clearance between Romeo's ankles. The step length is chosen in the range 0.15 to 0.20 $\mathrm{m}$, which corresponds to a $0.30-0.40 \mathrm{~m}$ displacement of the swing foot and a velocity of 0.83 to $1.1 \mathrm{~km} / \mathrm{h}$. A summary of the other adaptations is shown in the following table 1 . We approximate most periodic functions by a sinus to have a simple model that is infinitely differentiable.

Once we have adapted the human walking motion to the $n=31$ variables of Romeo, we tried to run it on the robot model. Due to differences in dynamics, the ZMP position resulting from these trajectories will not satisfy 
Table 1 Main parameters of the trajectories for Romeo

\begin{tabular}{|c|c|c|c|c|}
\hline Variable & Period & Mean Value & Magnitude & Phase \\
\hline $\begin{array}{l}\text { Motion along } Z \\
\text { of the CoM }\end{array}$ & $T$ & 1.12 (leg length) & $\begin{array}{l}\text { about } 2 \% \\
\text { of height }\end{array}$ & $\begin{array}{l}\text { Minimum in } \\
\text { middle of DS }\end{array}$ \\
\hline $\begin{array}{l}\text { Motion along } X \\
\text { of the CoM }\end{array}$ & $\begin{array}{l}\text { linear } \\
\text { progression }\end{array}$ & - & - & - \\
\hline $\begin{array}{l}\text { Motion along } Y \\
\text { of the CoM }\end{array}$ & $T$ & 0 & $\begin{array}{l}\text { about } 2 \% \\
\text { of height }\end{array}$ & $\begin{array}{l}\text { Zero around } \\
80 \% \text { of DS }\end{array}$ \\
\hline Trunk roll & $T$ & 0 & $5^{\circ}$ & $\begin{array}{l}\text { Minimum in } \\
\text { middle of DS }\end{array}$ \\
\hline Trunk pitch & $T$ & $6^{\circ}$ & $2^{\circ}$ & $\begin{array}{l}\text { Maximum } \\
\text { at beginning } \\
\text { and end of DS }\end{array}$ \\
\hline $\begin{array}{l}\text { Swing foot } \\
\text { height }\end{array}$ & cycloid $2 \mathrm{~T}$ & - & & \\
\hline $\begin{array}{l}\text { Swing foot } \\
\text { pitch }\end{array}$ & cycloid $2 \mathrm{~T}$ & $\overline{-}$ & $-20^{\circ}$ to $81^{\circ}$ & $\begin{array}{l}\text { Minimum right } \\
\text { after impact. }\end{array}$ \\
\hline
\end{tabular}

the equilibrium condition. We checked that the ZMP trajectory is outside the support polygon, as is visible in figure 2

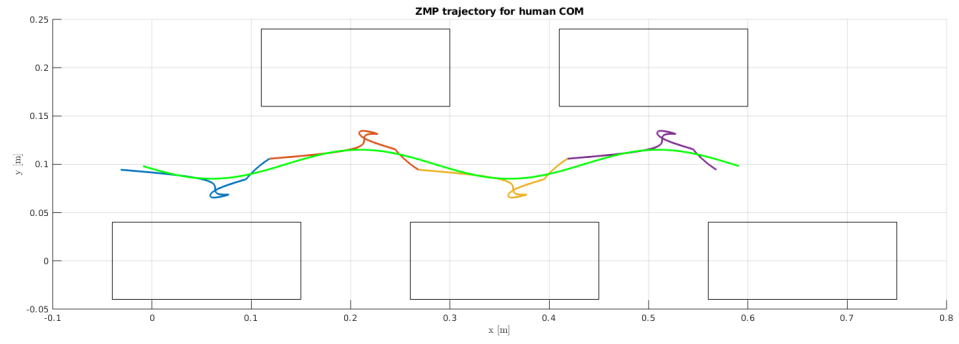

Fig. 2 ZMP (multicolored) obtained if the human COM motion (in green) is applied to the robot Romeo.

To solve this problem, we need to impose the ZMP trajectory instead of the CoM by using the Essential model first introduced in [5].

\section{Essential model}

Instead of imposing as many trajectories as there are degrees of freedom (DoFs), we will leave two DoFs to allow for a better placement of the ZMP. 
Since the relation between ZMP and CoM is considered as a determining feature of human gait $[2,15]$, and the positions of CoM and ZMP are strongly linked, we choose to "set free" the horizontal coordinates $r_{f}=(x, y)$ of CoM in order to adapt to the imposed trajectory of the ZMP.

To take inspiration from the human motion, let us introduce $r \in \mathbb{R}^{31 \times 1}$ :

$$
\begin{aligned}
r= & \left(r_{f}, r_{c}\right)^{\top}=\left(x, y, z(t), x_{f}(t), y_{f}(t), z_{f}(t), \psi_{f}(t),\right. \\
& \left.\theta_{f}(t), \phi_{f}(t), \psi_{t r}(t), \theta_{t r}(t), \phi_{t r}(t), q_{13}(t), \cdots, q_{31}(t)\right)^{\top} .
\end{aligned}
$$

We define $r_{c}$ as the vector of the 29 variables of $r$ for which the trajectories are imposed. $z(t)$ defines the desired altitude of the CoM. $x_{f}(t), y_{f}(t), z_{f}(t)$ and $\psi_{f}(t), \theta_{f}(t), \phi_{f}(t)$ describe the desired position and desired orientation of the free foot, and $\left(\psi_{t r}, \theta_{t r}, \phi_{t r}\right)$ give the desired orientation of the torso link. The upper-body variable joints are defined by $q_{13}$ to $q_{31}$. The desired motion for $r_{c}(t)$ is defined based on human motion as summarized in table 1 .

The robot configuration can be defined by the vector $q$ or $r$ and a geometric model can be built. Let $q=g\left(r_{f}, r_{c}\right), \dot{q}$ and $\ddot{q}$ are deduced thanks to the kinematic models as follows:

$$
\dot{q}=J_{f} \dot{r}_{f}+J_{c} \dot{r}_{c}, \quad \ddot{q}=J_{f} \ddot{r}_{f}+\dot{J}_{f} \dot{r}_{f}^{2}+J_{c} \ddot{r}_{c}+\dot{J}_{c} \dot{r}_{c}^{2} .
$$

Here $J_{f} \in \mathbb{R}^{31 \times 2}$ and $J_{c} \in \mathbb{R}^{31 \times 29}$. In the current study the evolution of $r_{c}$ is chosen as a function of time, thus the joint evolution can be expressed as function of $r_{f}, \dot{r}_{f}, \ddot{r}_{f}$ and t only :

$$
q=g_{t}\left(r_{f}, t\right), \quad \dot{q}=J_{f} \dot{r}_{f}+v\left(t, r_{f}\right), \quad \ddot{q}=J_{f} \ddot{r}_{f}+\dot{J}_{f} \dot{r}_{f}^{2}+a\left(t, r_{f}, \dot{r}_{f}\right) .
$$

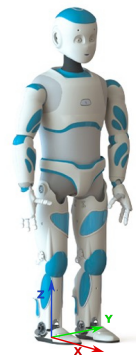

a)

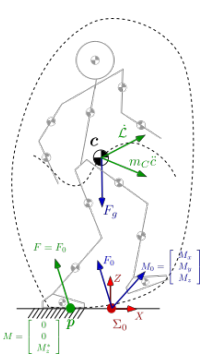

b)

Fig. 3 a) Photography of Romeo. b) Illustration of the global equilibrium.

To evaluate the feasibility of a walking trajectory, it is necessary to calculate the effects of external forces acting on the humanoid robots. The origins of these external forces are the gravity force $\boldsymbol{F}_{g}$ and the ground reaction forces acting applied on each foot figure $3 \mathrm{~b}$ ). The resulting ef- 
fect of the ground reaction is defined by the wrench $\in \mathbb{R}^{6 \times 1}\left(\boldsymbol{F}_{0}, \boldsymbol{M}_{0}\right)^{\top}=$ $\left(F_{x}, F_{y}, F_{z}, M_{x}, M_{y}, M_{z}\right)^{\top}$ in a reference frame $\Sigma_{0}$. The global equilibrium of the robot can be written as:

$$
\left(\begin{array}{c}
\boldsymbol{F}_{0} \\
\boldsymbol{M}_{0}
\end{array}\right)=\left(\begin{array}{c}
A_{F} \\
A_{M}
\end{array}\right) \ddot{q}+\left(\begin{array}{c}
d_{F}(q, \dot{q}) \\
d_{M}(q, \dot{q})
\end{array}\right)
$$

where $q \in \mathbb{R}^{31 \times 1}$ is the joint vector of the robot.

Using equation (3), the global equilibrium (4) can be rewritten:

$$
\left(\begin{array}{c}
\boldsymbol{F}_{0} \\
\boldsymbol{M}_{0}
\end{array}\right)=\left(\begin{array}{c}
A_{F r}\left(t, r_{f}\right) \\
A_{M r}\left(t, r_{f}\right)
\end{array}\right) \ddot{r}_{f}+\left(\begin{array}{c}
d_{F r}\left(t, r_{f}, \dot{r}_{f}\right) \\
d_{M r}\left(t, r_{f}, \dot{r}_{f}\right)
\end{array}\right)
$$

Let $\boldsymbol{p}=\left(p_{x}, p_{y}, 0\right)^{\top}$ be the global zero moment point (ZMP). Its coordinates $p_{x}$ and $p_{y}$ satisfy :

$$
F_{z} p_{x}+M_{y}=0, F_{z} p_{y}-M_{x}=0
$$

$\left(p_{x}, p_{y}\right)$ must be inside the convex hull of support for all times in order to satisfy the dynamic equilibrium condition [16]. To be sure to find a periodic motion that satisfies the equilibrium condition, we choose a desired evolution $\left(p_{x}(t), p_{y}(t)\right)$ of the ZMP. During the SS phase the desired motion of the $\mathrm{ZMP}$ is a function of time to define a migration of the ZMP from the heel to the toe of the stance foot. In DS phase the desired motion of the ZMP is defined by a linear evolution form the final position of the ZMP at the end of the SS phase on the stance foot, until the initial position of the ZMP at the beginning of the SS on the next stance foot. Using equation (6), and using the $3^{\text {th }}, 4^{\text {th }}$ and $5^{\text {th }}$ lines of (5), we obtain :

$$
\begin{aligned}
& \left(A_{F r z}\left(t, r_{f}\right) \ddot{r}_{f}+d_{F r z}\left(t, r_{f}, \dot{r}_{f}\right)\right) p_{x}(t)+A_{M r y}\left(t, r_{f}\right) \ddot{r}_{f}+d_{M r y}\left(t, r_{f}, \dot{r}_{f}\right)=0 \\
& \left(A_{F r z}\left(t, r_{f}\right) \ddot{r}_{f}+d_{F r z}\left(t, r_{f}, \dot{r}_{f}\right)\right) p_{x}(t)-A_{M r x}\left(t, r_{f}\right) \ddot{r}_{f}-d_{M r x}\left(t, r_{f}, \dot{r}_{f}\right)=0
\end{aligned}
$$

that isolates the essential characteristic of the walking that is the relationship between the ZMP and the CoM. Solving of equation (7) gives the Essential model describing the acceleration of the horizontal positions $x$ and $y$ of the CoM, that are defined to achieve to an imposed evolution of the ZMP:

$$
\ddot{r}_{f}=f\left(r_{f}, \dot{r}_{f}, t, p_{x}(t), p_{y}(t)\right) .
$$

By integration of (8) from initial conditions we can calculate the current values of $\dot{r}_{f}$, i.e $\dot{x}, \dot{y}$, and $r_{f}$, i.e $x$, and $y$. To sum up, the evolution of $x$ and $y$ is not imposed in order to allow them to adapt to the imposed evolution of the ZMP. With this strategy to define a reference trajectory of walking, which is based on the Essential model (8) and $r_{c}(t)$, no approximations are made to the dynamic model when designing the humanoid walking. The method ensures the feasibility of a walking trajectory from the point of view of the condition on the ZMP. The choice of $z(t)$ of the CoM allows to satisfy the 
positivity of the vertical component of the resultant ground reaction force during the walking. The condition of no slipping can be checked based on the knowledge of $\ddot{r}_{f}$ and $\ddot{z}$.

Then the torques required to produce the motion have to be calculated. During the SS phase, considering the stance foot motionless on the ground, we can define the dynamic behavior of the robot:

$$
\tau=A_{r}\left(t, r_{f}\right) \ddot{r}_{f}+d_{r}\left(t, r_{f}, \dot{r}_{f}\right)
$$

In DS phase effort $\left(\begin{array}{c}F_{\text {ext }} \\ M_{\text {ext }}\end{array}\right)$ are applied on the second leg, (9) becomes

$$
\tau=A_{r}\left(t, r_{f}\right) \ddot{r}_{f}+d_{r}\left(t, r_{f}, \dot{r}_{f}\right)+J_{e x t}\left(\begin{array}{c}
F_{e x t} \\
M_{e x t}
\end{array}\right) .
$$

The global equation gives the global reaction force $\boldsymbol{F}_{0}, \boldsymbol{M}_{0}$, but the distribution on both legs is free and will modify the actuation torque. During double support, the global ZMP is the barycentre of the two local ZMPs on each foot, this implies that the global ZMP and the local ZMPs are aligned. The evolution of the global ZMP is chosen in order that during all the DS, the two local ZMPs keep a constant pose corresponding to the final pose of the ZMP in SS : $p_{5}$, and the initial pose of the ZMP for the next SS $: p_{2}$. We can calculate the vertical reaction force on leg 1 and $2 F_{1 z}$ and $F_{2 z}$ by solving this system:

$$
\begin{aligned}
& \frac{p_{1 x} F_{1 z}+p_{2 x} F_{2 z}}{F_{1 z}+F_{2 z}}=p_{x} \\
& \frac{p_{1 y} F_{1 z}+p_{2 y} F_{2 z}}{F_{1 z}+F_{2 z}}=p_{y}
\end{aligned}
$$

To avoid slipping, the ratio between tangential and normal force for the global equilibrium is conserved for each leg. The components $F_{1 x}, F_{1 y}, F_{2 x}$, and $F_{2 y}$ are calculated to satisfy:

$$
\begin{aligned}
& \frac{F_{1 x}}{F_{1 z}}=\frac{F_{2 x}}{F_{2 z}}=\frac{F_{1 x}+F_{2 x}}{F_{1 z}+F_{2 z}} \\
& \frac{F_{1 y}}{F_{1 z}}=\frac{F_{2 y}}{F_{2 z}}=\frac{F_{1 y}+F_{2 y}}{F_{1 z}+F_{2 z}}
\end{aligned}
$$

By using (11) and (12) we find $M_{z}=M_{1 z}+M_{2 z}$. The moment around the $z$ axis is also share between the two legs using a similarly distribution to the force components (12) as follows:

$$
\frac{M_{1 z}}{F_{1 z}}=\frac{M_{2 z}}{F_{2 z}}=\frac{M_{1 z}+M_{2 z}}{F_{1 z}+F_{2 z}}
$$




\section{Periodic walking}

The target walking motion is periodic, with a step that is composed of SS phase and a DS phase. There is no impact at the end of the SS phase. To find the walking motion a boundary problem is solved as follows. The algorithm starts from an initial guess of CoM position and velocity $\left(x\left(t_{0}\right), y\left(t_{0}\right), \dot{x}\left(t_{0}\right), \dot{y}\left(t_{0}\right)\right)$ at the start of DS phase. The condition for periodicity is

$$
\left(x\left(t_{0}\right), y\left(t_{0}\right), \dot{x}\left(t_{0}\right), \dot{y}\left(t_{0}\right)\right)=\left(x\left(t_{0}+T\right), y\left(t_{0}+T\right), \dot{x}\left(t_{0}+T\right), \dot{y}\left(t_{0}+T\right)\right)
$$

tacking into account the change of the reference frame when the two legs switch their role just after the end of the current step. So $\dot{x}\left(t_{0}+T\right), \dot{y}\left(t_{0}+T\right)$ are the initial velocities of the CoM in DS of the next step. The boundary value problem is, what are $x\left(t_{0}\right), y\left(t_{0}\right), \dot{x}\left(t_{0}\right)$ and $\dot{y}\left(t_{0}\right)$ such that after integration of (8) over the time interval $\left[t_{0}, t_{0}+T\right]$ the cyclic condition (14) is satisfied.

\section{Numerical results}

We obtained a cyclic trajectory for a step size of $0.15 \mathrm{~m}$, all other parameters being the same as described in table 1. A stick-diagram over one cycle of this cyclic walking motion is presented figure 4 .

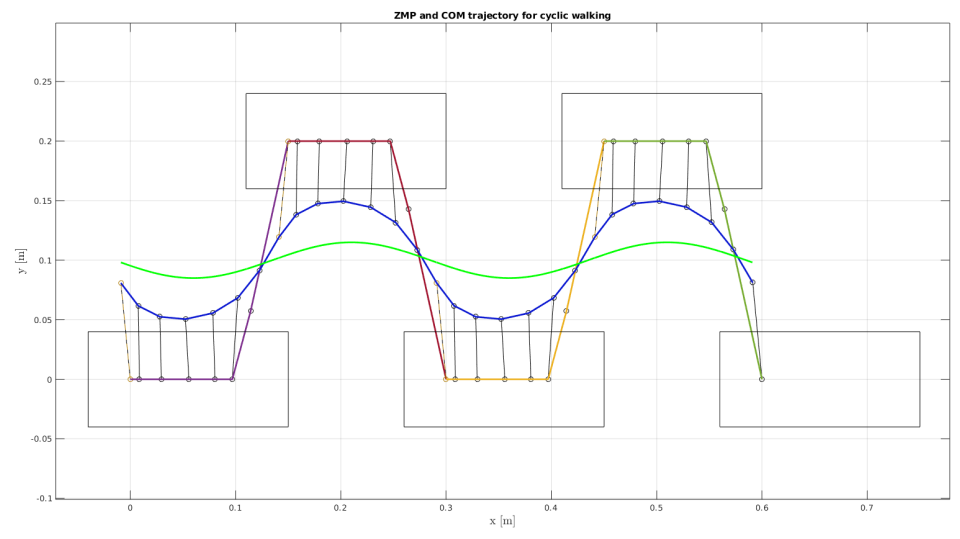

Fig. 4 Obtained COM trajectory (in blue) for the imposed human-like ZMP trajectory (multicolored), compared to a typical human COM trajectory (in green). 


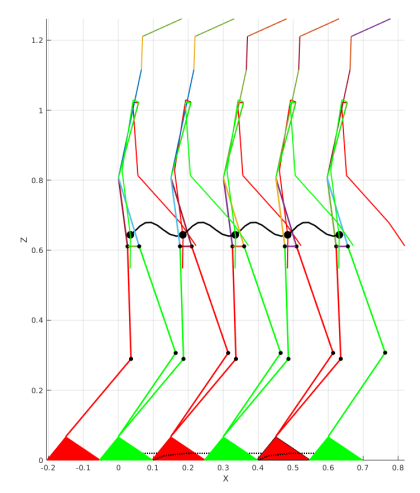

Fig. 5 Stick-diagram of a walking.

We observe on figure 4 that the COM trajectory is oscillating in the $Y$ direction a lot more than what is typical for human walking. This difference can be explained by the slightly larger step width (necessary because of geometric constraints on Romeo) and the overall slower walking velocity. Indeed, the slower the walking gait, the closer it gets to semi static, and the larger the COM oscillations in $Y$ direction [7].

This result can be compared to the "raw" ZMP trajectory based on a human-like COM trajectory without constraints on the ZMP, as presented on figure 2. It is obvious that this trajectory would not be viable, as the ZMP trajectory is outside of the convex hull of the foot during single support phase. This proves the relevance of the approach used with the Essential Model, imposing constraints on the ZMP position rather than COM allows to achieve a dynamically stable walking motion.

\section{Effect of ZMP evolution on torques}

The results presented above correspond to an evolution of the ZMP going from the heel to the tip of each foot (see figure 4). The torque at the ankle is directly affected by the pose of the ZMP. It can be seen in figure $6\left(2^{\text {nd }}\right.$ image), that the propulsive torque is low at the beginning of the step. As a consequence, a high propulsive torque is required at the knee joint (figure 6 ( $3^{\text {th }}$ image). In fact this high torque exceeds the limits of the actuator (shown in dotted line) of the robot Romeo. We explored the effect of the influence of ZMP evolution. The results show that a modification of the ZMP trajectory influences the torques in the support knee and in the support ankle. A ZMP that has a constant position in front of the foot allows a higher propulsive force at the beginning of the SS, and thus allows to decrease the propulsive 
force at knee, and then produce a knee torque compatible with the actuator of Romeo.

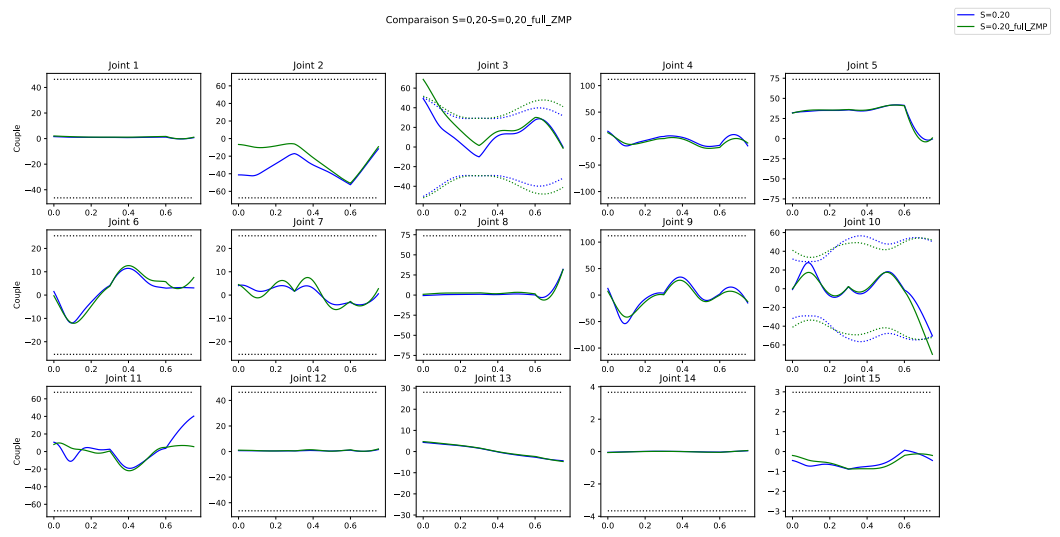

Fig. 6 Joint torques (N.m) versus time (s): comparison of the torque in the lower part of the robot for two cyclic trajectories with a step size of $0.20 \mathrm{~m}$ and a period of $0.75 \mathrm{~s}$. The trajectory in green is with a human like ZMP evolution in DS, and the trajectory in blue has a ZMP constrained to the front of the foot.

\section{Conclusions}

We developed a 3D cyclic walking motion for a humanoid robot, Romeo. Each step is composed of a SS phase and a DS phase. The design is based on the use of the Essential model that ensures the feasibility of the motion by satisfying the ZMP condition, which is the hardest constraint to meet. The other generalized variables of the robot are defined as smooth periodic functions of time taking inspiration from human walking motions. A dynamic model and the global equilibrium of the robot prove that the obtained walking motions are valid. The perspectives are to complete the cyclic walking motions with a starting phase and a stopping phase and to test a set of walking motions with an experimental platform. 


\section{References}

1. D. Tlalolini, Y. Aoustin, and C. Chevallereau, "Design of a walking cyclic gait with single support phases and impacts for the locomotor system of a thirteenlink 3d biped using the parametric optimization," Multibody System Dynamics, vol. 23 , no. 1 , pp. 33-56, 2010.

2. S. Kajita, H. Hirukawa, K. Harada, and K. Yokoi, Introduction to humanoid robotics. Springer, 2014, vol. 101.

3. A. D. Ames, E. A. Cousineau, and M. J. Powell, "Dynamically stable bipedal robotic walking with nao via human-inspired hybrid zero dynamics," in Proc. of the 15th ACM int. conf. on Hybrid Systems: Computation and Control. ACM, 2012, pp. 135-144.

4. S. Sakka, Imitation des mouvements humains par un robot humanö̈de sous contrainte d'équilibre. HDR, Université Pierre et Marie Curie (UPMC), 2017.

5. V. De-León-Gómez, Q. Luo, A. Kalouguine, J. A. Pámanes, Y. Aoustin, and C. Chevallereau, "An essential model for generating walking motions for humanoid robots," Robotics and Autonomous Systems, vol. 112, pp. 229-243, 2019.

6. J. Rose and J. G. Gamble, Human walking, 3rd ed. Williams \& Wilkins, 2006.

7. M. S. Orendurff, A. D. Segal, G. K. Klute, J. S. Berge, E. S. Rohr, and N. J. Kadel, "The effect of walking speed on center of mass displacement." Journal of Rehabilitation Research \& Development, vol. 41, no. 6, 2004.

8. T. Jurcevic Lulic and O. Muftic, "Trajectory of the human body mass centre during walking at different speed," in DS 30: Proc. of DESIGN 2002, the 7th Int. Design Conf., Dubrovnik, 2002.

9. M. Grundy, P. Tosh, R. McLeish, and L. Smidt, "An investigation of the centres of pressure under the foot while walking," J. of bone and joint surgery. British volume, vol. 57, no. 1, pp. 98-103, 1975.

10. P. Sardain and G. Bessonnet, "Zero moment point-measurements from a human walker wearing robot feet as shoes," IEEE Trans. on Systems, Man, and Cybernetics-Part A: Systems and Humans, vol. 34, no. 5, pp. 638-648, 2004.

11. A. Thorstensson, J. Nilsson, H. Carlson, and M. R. ZOMLEFER, "Trunk movements in human locomotion," Acta Physiologica Scandinavica, vol. 121, no. 1, pp. 9-22, 1984.

12. P. Meyns, S. M. Bruijn, and J. Duysens, "The how and why of arm swing during human walking," Gait \&s posture, vol. 38, no. 4, pp. 555-562, 2013.

13. Y. Aoustin and A. M. Formalskii, "3d walking biped: optimal swing of the arms," Multibody System Dynamics, vol. 32, no. 1, DOI 10.1007/s11044-013-9378-3, pp. 55-66, 2014.

14. K. T., C. Chevallereau, and A. Y., "Effect of circular arc feet on a control law for a biped," Robotica, vol. 27, no. 4, pp. 621-632, 2008.

15. T. Koolen, T. de Boer, J. Rebula, A. Goswami, and J. Pratt, "Capturabilitybased analysis and control of legged locomotion, part 1: Theory and application to three simple gait models," Int. J. of Robotics Research, vol. 31, no. 09, pp. 1094-1113, 2012.

16. M. Vukobratovic and B. Borovac, "Zero-moment point-thirty five years of its life," Int. J. of Humanoid Robotics, vol. 1, no. 1, pp. 157-173, 2004. 